\title{
Novel Approach to Microbiological Study of Chronic Inflammations at Upper Respiratory Tract: Research of Blood L-Form Microbiota
}

\author{
Nadya Markova \\ Institute of Microbiology, Bulgarian Academy of Sciences, Sofia, Bulgaria \\ Email: nadya.markova@gmail.com
}

How to cite this paper: Markova, N. (2021) Novel Approach to Microbiological Study of Chronic Inflammations at Upper Respiratory Tract: Research of Blood L-Form Microbiota. Open Journal of Medical Microbiology, 11, 144-156.

https://doi.org/10.4236/ojmm.2021.113012

Received: July 2, 2021

Accepted: August 3, 2021

Published: August 6, 2021

Copyright $\odot 2021$ by author(s) and Scientific Research Publishing Inc. This work is licensed under the Creative Commons Attribution International License (CC BY 4.0).

http://creativecommons.org/licenses/by/4.0/

\begin{abstract}
Background: The recognition of human blood microbiota, consisting of cell wall-deficient microbes (L-forms), is a major challenge today in the field of microbiology. There are accumulating data confirming the concept of "internal" blood L-form microbiota and its significance for health and diseases. Finding out whether the blood microbiota can be of diagnostic and prognostic importance for detection and evaluation of chronic infections anywhere in the body is a major objective. In the context of chronically infected upper respiratory tract (URT), the aim of the current study was to understand whether a local infection can be a source for entry of bacteria and fungi in the blood. Methods: Blood samples from six persons with chronic inflammations in URT diagnosed with hypertrophied adenoids, chronic sinusitis, nasal polyps, chronic naso-pharyngitis and one control healthy person were studied. Blood microbiota assessment methodology that be used, included three phases: 1) isolation of L-form cultures from blood-development and propagation; 2) cultivation directed to conversion of L-forms into bacterial and fungal cultures; 3) isolation of pure classical bacterial and fungal cultures and their identification by MALDI-TOF method. Results: From the patients were isolated L-forms of opportunistic bacteria (Streptococcus mitis, Roseomonas mucosa, Dermacoccus nishinomiyaensis, Enterococcus faecalis, Acinetobacter johnsonii, Pseudomonas putida, Staphylococcus aureus, Pseudomonas luteola, Enterobacter cloacae) and fungi such as Rhodotorula mucilaginosa, Aspergillus niger, Aspergillus fumigatus and Mucorales. Conclusion: The novel innovative methodology for assessment of blood L-form microbiota was successfully applied for detection of microbes responsible for chronic infections at URT.
\end{abstract}

\section{Keywords}

L-Forms, Blood Microbiota, Chronic Inflammation, Upper Respiratory Tract 


\section{Introduction}

On the basis of generated knowledge about the unique nature of L-forms (cell wall-deficient microbe variants) and the accumulated data by different authors about presence/persistence of L-forms in human blood, a concept was formulated, of "internal" blood L-form microbiota [1]-[9]. The hypothesis of blood L-form microbiota has been confirmed by several studies which led to novel insights with relevance to the role of persisting microbes in some chronic (noncommunicable) diseases [10] [11] [12] [13] [14]. The use of original methodology for cultivating of L-forms provides us the opportunity to determine their identity i.e. which species of bacteria or fungi they belong to. It has been found that the blood of healthy people is habited by bacterial L-forms, but the invasion of L-forms of opportunistic bacteria and fungi in blood of people with health problems can lead to dysbiosis [13] [14]. It is believed that the sources for entry of opportunistic bacteria and fungi into blood can be a dysbiotic microbial associations (microbiota) at various anatomical sites in the body (intestine, upper respiratory tract, skin, urogenital tract) contacting with the external environment. Microbial translocation from places with normal microbiota into the blood can be a result of chronic inflammation and impaired mucosal protective/barrier functions, respectively the increased mucosal permeability [15]. Potgieter et al. suggest that despite the physical barrier between external and internal environments, conditions could be established that might permit microbial entry in blood [15]. The current study is aimed to evaluate how blood L-form microbiota can mirror a state of dysbiotic microbiota in upper respiratory tract of patients with chronic inflammations and to be a criterion for disrupted mucosal barrier function.

\section{Methods}

Patients: Blood samples from seven persons (male and female individuals of varying ages) were studied as listed in Table 1 . The investigated individuals were with chronic inflammations in sino-nasal cavities with diagnoses-adenoid hypertrophy, chronic sinusitis, nasal polyps, chronic nasopharyngitis. Some of them were with complicating diseases such as asthma, cervical lymphadenitis and pneumonia (rare case of "round" pneumonia). One control healthy person was studied as well. From all investigated persons were taken aseptically venous blood samples using K2E-EDTA Vacutainer tubes (BD Vacutainer, Plymouth, UK). Informed consent for the use of the blood samples for research purposes was obtained from all participants and/or their legal guardians, respectively. All blood samples were handled and anonymized, according to the national ethical and legal guidelines, while the study protocol was approved by the Ethics Committee of Scientific Studies Involving Human Experimentation at the Medical University of Sofia.

Blood microbiota assessment (BMA): The methodology of blood microbiota assessment involved three successive phases: 1 ) isolation of L-form cultures from 
Table 1. Isolation of bacterial and fungal cultures from blood of patients with inflammations at upper respiratory tract.

\begin{tabular}{|c|c|c|c|c|}
\hline \multirow{2}{*}{$\begin{array}{c}\text { Patient } \\
\text { Gender/age }\end{array}$} & \multirow[t]{2}{*}{ Diagnosis } & \multirow[t]{2}{*}{ Bacterial isolates } & \multicolumn{2}{|c|}{ Fungal isolates } \\
\hline & & & Yeasts & Filamentous fungi \\
\hline 1. Male/7y.o. & Adenoid vegetation & $\begin{array}{l}\text {-Streptococcus mitis } \\
\text {-Roseomonas mucosa } \\
\text {-Dermacoccus nishinomiyaensis }\end{array}$ & Rhodotorula mucilaginosa & -Aspergillus fumigatus \\
\hline 2. Fmale/32y.o & Chronic sinusitis + migraine & Enterococcus faecalis & Rhodotorula mucilaginosa & $\begin{array}{l}\text {-Mucorales } \\
\text {-Aspergillus niger }\end{array}$ \\
\hline 3. Fmale/34y.o & Nasal polyps + asthma bronchialae & Acinetobacter johnsonii & Rhodotorula mucilaginosa & Aspergillus fumigatus \\
\hline 4. Male/68y.o & $\begin{array}{l}\text { Chronic sinusitis + cervical } \\
\text { lymphadenopathy }\end{array}$ & $\begin{array}{l}\text {-Enterococcus faecalis } \\
\text {-Pseudomonas putida, }\end{array}$ & Rhodotorula mucilaginosa & - \\
\hline 5. Fmale/32y.o. & Chronic nasopharyngitis & -Staphylococcus aureus & - & Aspergillus fumigatus \\
\hline 6. Fmale/62y.o. & $\begin{array}{l}\text { Upper respiratory tract inflammation } \\
\text { + "round" pneumonia }\end{array}$ & $\begin{array}{l}\text {-Pseudomonas luteola } \\
\text {-Enterobacter cloacae }\end{array}$ & Rhodotorula mucilaginosa & - \\
\hline 7. Fmale/34y.o. & Control/healthy person & - & - & - \\
\hline
\end{tabular}

Blood-development and propagation; 2) cultivation to direct conversion of Lforms into bacterial and fungal cultures; 3) isolation and obtainment of pure classical bacterial and fungal cultures, as well as their identification via MALDITOF method.

Isolation of L-form cultures-development and propagation: As described in our previous studies, two protocols designated as "classical" and "filtration" were used for the isolation of microbial L-type cultures from blood samples [10] [11] [13]. In short, the blood sample was cultivated after a procedure of lysis with sterile distilled water at strictly fixed $\mathrm{v} / \mathrm{v}$ ratio and after 30 minutes of exposure to room temperature. "Classical" and "filtration" protocols were described in previous study as follows [13]. As per the "classical" protocol (CL), the aliquots from lysed blood samples were inoculated in tubes with Tryptic Soy Broth (TSB, Becton Dickinson) and incubated at $37^{\circ} \mathrm{C}$ for 72 hours. As for the "filtration" protocol (F), after inoculation TSB was filtered through a bacterial filter with $0.2 \mu \mathrm{m}$ pore size and was also incubated at $37^{\circ} \mathrm{C}$ for 72 hours.

Cultivation directed to conversion of $\mathrm{L}$-forms into bacterial and fungal cultures: Strictly fixed aliquots from primary broths (CL and F) were sub-cultured again in three variants of broth media (TSB, TSB with Gentamycin of $100 \mu \mathrm{g} / \mathrm{ml}$ and Sabouraud Dextrose broth-SDB with Chloramphenicol of $50 \mu \mathrm{g} / \mathrm{ml}$ ) and parallel plated on three variant of semisolid media -TSA, TSA with Gentamycin of $100 \mu \mathrm{g} / \mathrm{ml}$ and Sabouraud Dextrose Agar-SDA with Chloramphenicol of 50 $\mu \mathrm{g} / \mathrm{ml}$. The semisolid media were solidified with $0.8 \%$ (w/v) Agar (Fluca). TSB and TSA were incubated at $37^{\circ} \mathrm{C}$, while SDB and SDA at $25^{\circ} \mathrm{C}$. Passages in broth and semisolid media were performed using technique described in previous study [3]. In control experiments for the sterile performance of the technical procedures, broths and semisolid media were inoculated with sterile saline and subsequent transfers were done by the same technique. All cultures were period- 
ically observed for appearance of growth and morphological transformations within 2 months. Direct light microscopic observations of native preparations from cultures were combined with Gram and Giemsa stained smears.

Identification of isolated pure classical bacterial and fungal cultures by MALDI-TOF method: The isolated pure bacterial and yeast cultures were identified by matrix assisted laser desorption ionization-time of flight mass spectrometry technology (MALDI-TOF MS) using intact cells. This tool for microbial (bacteria and fungi) identification is based on automation in proteomics technology. Identification of microbes by MALDI-TOF MS is done by comparing the characteristic spectrum called peptide mass fingerprints (PMF) of unknown organisms with the PMFs contained in the database. This technology is already applied worldwide for microbial identification, by using commercial libraries of organisms PMFs [16]. MALDI-TOF MS equipment (Vitek MS-BIOMERIEUX) was used in the current study and all procedures were done according to the protocol of the manufacturer by trained laboratory personnel in a national public health laboratory.

\section{Results}

L-form cultures were isolated from blood of all investigated people. As seen in Table 1, from the six patients (no1-6) were recovered through reversion of Lforms during cultivation in broth, opportunistic bacterial species such as Streptococcus mitis, Roseomonas mucosa Dermacoccus nishinomiyaensis, Enterococcus faecalis, Acinetobacter johnsonii, Pseudomonas putida, Staphylococcus aureus, Pseudomonas luteola, Enterobacter cloacae and fungal species such as Rhodotorula mucilaginosa, Aspergillus niger, Aspergillus fumigatus and Mucorales. As described in previous study as well [13], the so called "non-cultivable elements" from blood started to develop as replicable L-forms (cell wall-deficient variants) in broth by using special techniques and intervals of sub-cultivations. Figure 1 demonstrates development of spherical and granular L-bodies with typical characteristics during the early phase of blood cultivation in broth. L-bodies were seen intracellularly inside polymorphonuclear and mononuclear leucocytes, or extracellularly located. Subsequently, L-bodies started replicating and formed population of L-form culture (Figure 1(E)). After a phase of development and multiplication of L-forms, from them were recovered pure bacterial culture, a process also known as reversion of cell wall-deficient variants into classical bacterial forms. Thereafter, the isolated bacterial species were identified with/by MALDI-TOF technique. As presented in Figure 2 and Figure 3, spherical L-forms recovered their cell walls during cultivation in broth and after sub-cultivation on semisolid media were isolated pure cultures of Acinetobacter johnsonii from patient 3, as well as Roseomonas mucosa and Streptococcus mitis from patient 1. Analogical L-form conversion into normal bacteria were also observed in the isolation of other bacterial species-Dermacoccus nishinomiyaensis (patient 1), Enterococcus faecalis (patient 2 and patient 4), Pseudomonas putida (patient 4), Staphylococcus aureus (patient 5), Pseudomonas luteola 


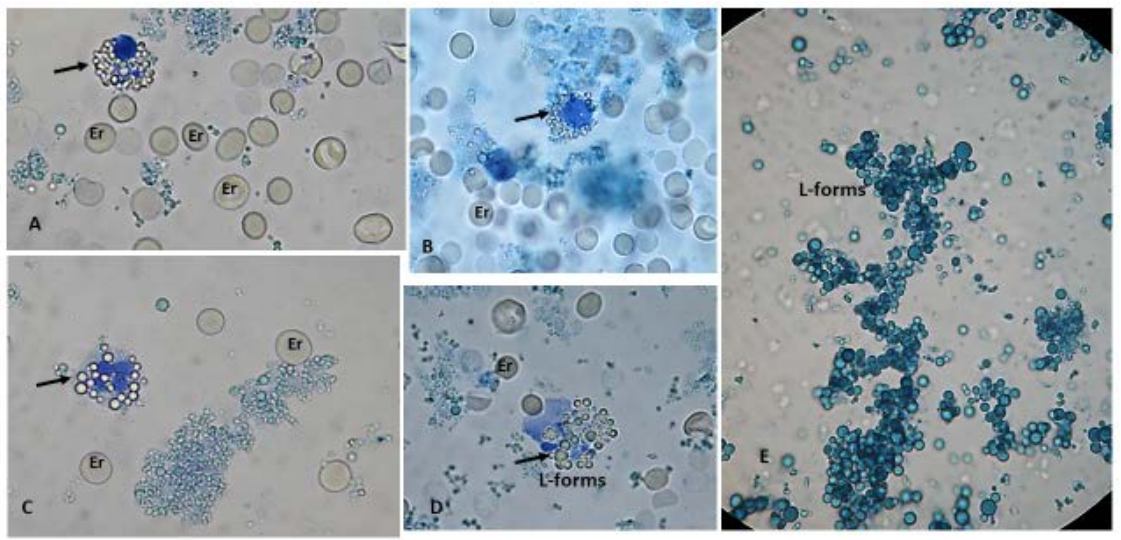

Figure 1. Development of L-form population in broth during cultivation of blood from patient No. 2 and No. 4. Spherical L-bodies, intracellularly seen in polymorphonuclear and mononuclear leucocytes, or extracellularly located (arrows). Native preparations with methylene blue. Er (erythrocytes). Magnification 1000×.
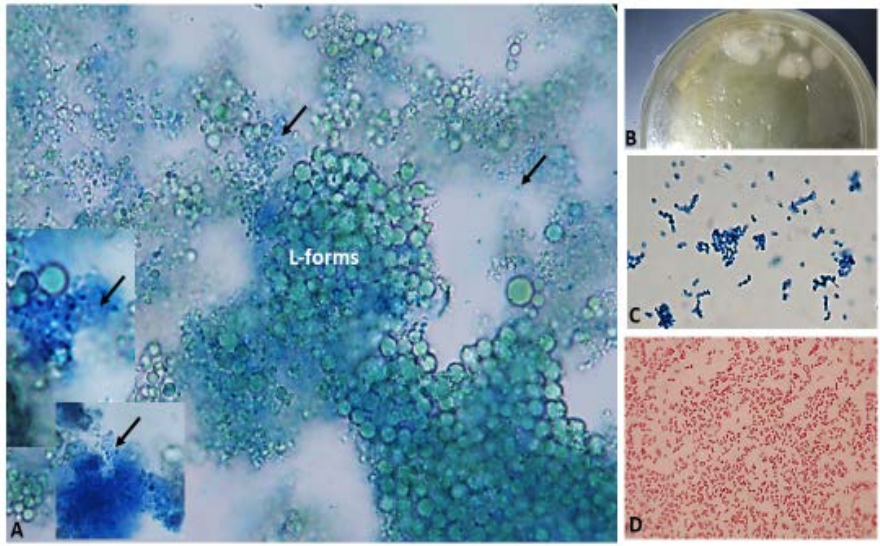

Figure 2. Transformation of L-forms into classical bacteria ((A) arrows) in broth and isolation of Acinetobacter johnsonii from blood of patient No. 3. (B) Colonies of Acinetobacter johnsonii; (C) Native preparation; (D) Gram stained smear. Magnification 1000×.

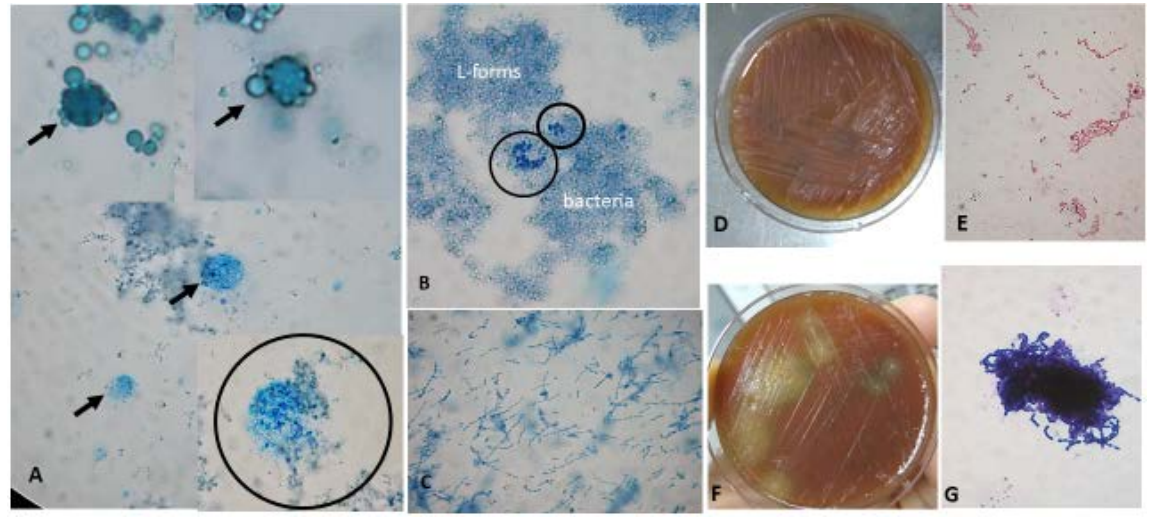

Figure 3. Transformation of L-forms into classical bacteria in broth (patient 1): (A) Spherical large L-bodies, some of them budding (arrows), others releasing reverted bacteria (circle); B. Granular L-forms and groups of reverted bacteria (circle); (C) Reverting bacteria-transitory forms. Isolation of Roseomonas mucosa (D); Gram stained smear (E) and of Streptococcus mitis (F); Gram stained smear (G). Magnification 1000×. 
patient 6), Enterobacter cloacae (patient 6). It is interesting to note that in 5 of the 6 investigated patients, were isolated L-forms of yeasts, identified as Rhodotorula mucilaginosa (Table 1). Fungal L-form conversion into classical yeast cells is presented in Figure 4. Large spherical L-bodies were seen when cultivating the blood initially in broth. Thereafter, fully reverted yeast cells were found and isolation of pure culture of Rhodotorula mucilaginosa became possible on solid medium. As seen in Figure 5, elements from life cycle of filamentous fungi (spherical bodies, fruiting bodies of cleistothecium type, germinating and tube-producing conidia and hyphae) were also observed in broths inoculated with blood. Mold cultures of Aspergillus fumigatus and Aspergillus niger were isolated as well. It should be noted that from blood of patient 2 was isolated
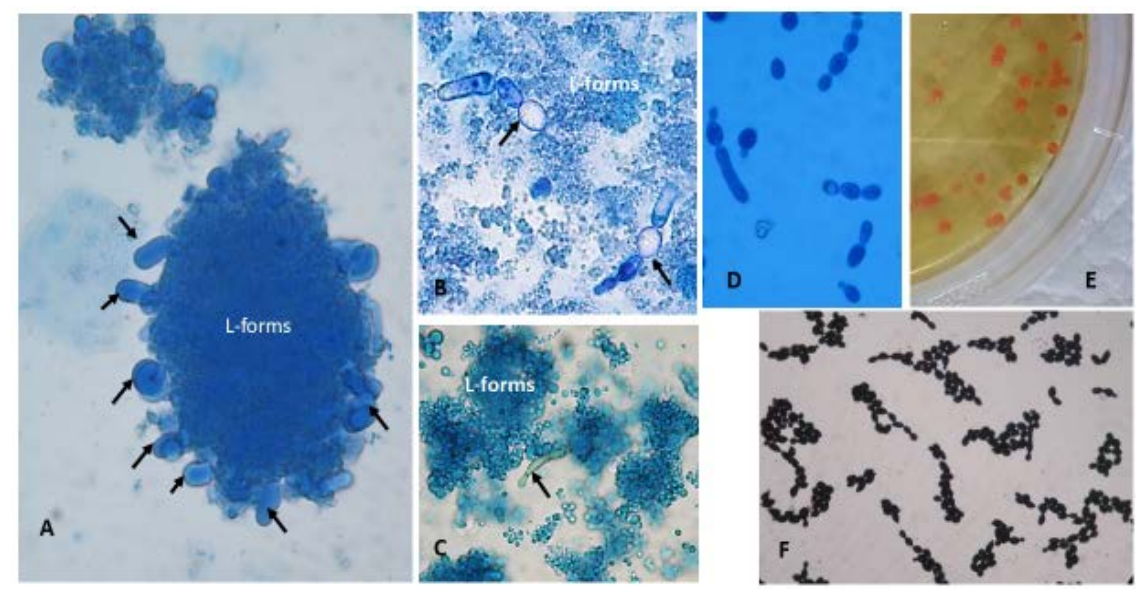

Figure 4. Transformation of fungal L-forms into classical yeast cells in broth (patient 1): (A) large spherical L-bodies (arrows); (B, C) granular L-forms and dividing yeast cells (arrows); (D) Fully recovered yeast cells; (E) Isolation of pure culture of Rhodotorula mucilaginosa; (F) Gram stained smear. Magnification 1000×.
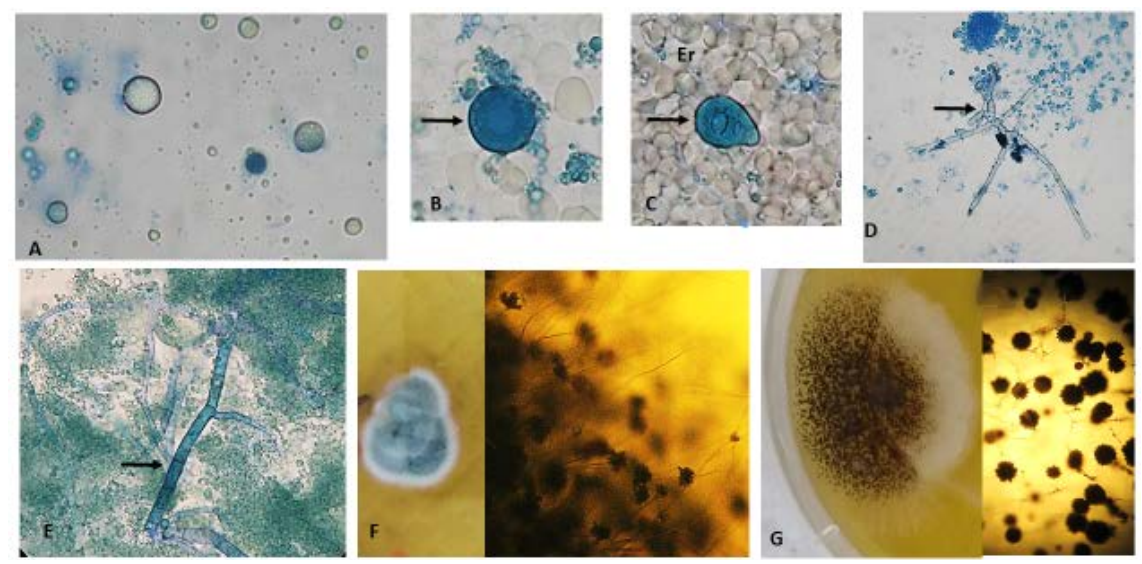

Figure 5. Observation of fungal elements from life cycle of filamentous fungi in SDBfrom blood of patients no1, 2, 3 and 5 (A-E): Spherical bodies/asci (A), Fruiting bodies of cleistothecium type (B, C); Germinating and tube-producing conidia (D); Formation of septate hyphae (E). After subsequent sub-cultivation on solid media (SDA), typical growth of Aspergillus fumigatus (F) and Aspergillus niger (G) and pictures of conidiophores. Magnification: (A-E)-1000×. (F, G)-200×. 
mold culture, recognized to belong to the order Mucorales on the basis of morphological characteristics (Figure 6). An interesting feature that was observed in patient 2 when culturing her blood in broth, was the production of mucoid extracellular secretes during the process of $\mathrm{L}$-form conversion into classical bacteria and fungi (Figure 7). What stood out in all patients was the isolation of co-associated polymicrobial communities consisting L-forms of bacteria and fungi. Figure 8 demonstrates isolation of such polymicrobial communities from patients 1, 3, 4 and 6 . It should be noted the observed unusual rough and strongly aggregated growth of such communities on semi-solid medium.

\section{Discussion}

The conception about "blood microbiota" in humans is presented in previous studies [1] [13] [14]. Two types of states are recognized-"eubiotic" vs. "dysbiotic" microbiota, that are of significance for health and disease. Last investigations of blood microbiota in patients with autism, multiple sclerosis (MS) and Parkinson's disease showed that cell wall-deficient variants (L-forms) of opportunistic bacteria and fungi may have a disease-trigger potential [14]. An interesting issue to address is, whether the blood microbiota can be of diagnostic and prognostic importance for detection and evaluation of chronic and latent (hidden)
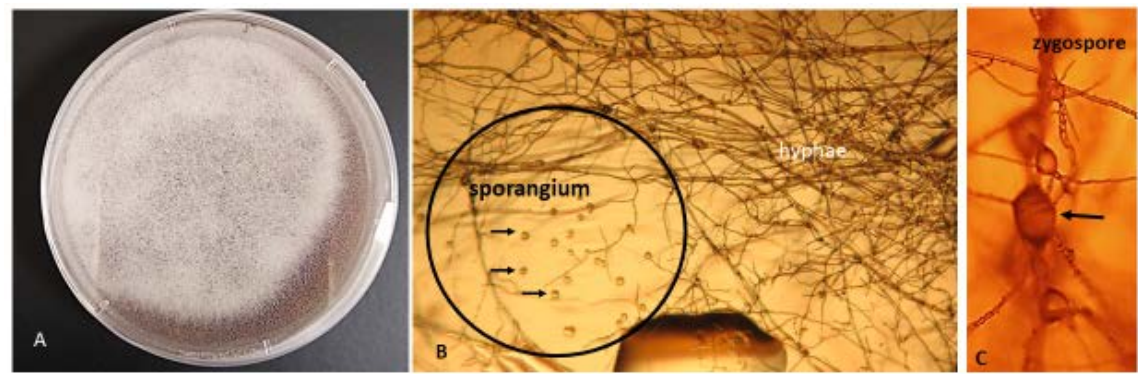

Figure 6. Isolation of mold culture from blood of patient 2, recognized as Mucorales (order) on the basis of morphological characteristics. (A) Mold growth; (B) Sporangium and hyphae; (C) Zygospore (arrow). Magnification: (B, C) - 200×.
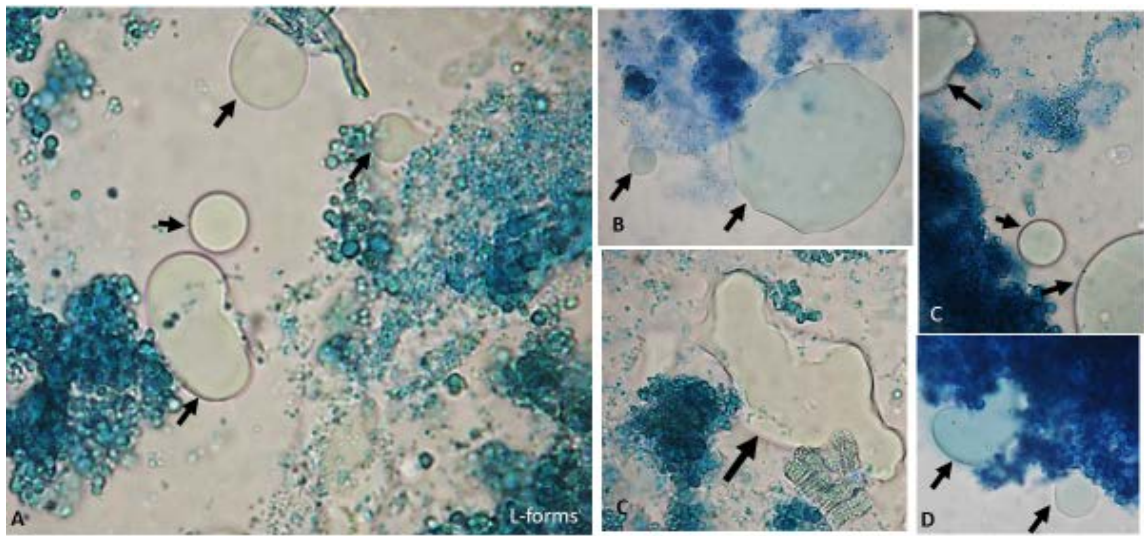

Figure 7. Secretion of mucoid fluids (arrows) during the process of L-form conversion into classical bacteria and fungi in patient 2. Native preparations from broth contrasted with methylene blue. Magnification: $1000 \times$. 

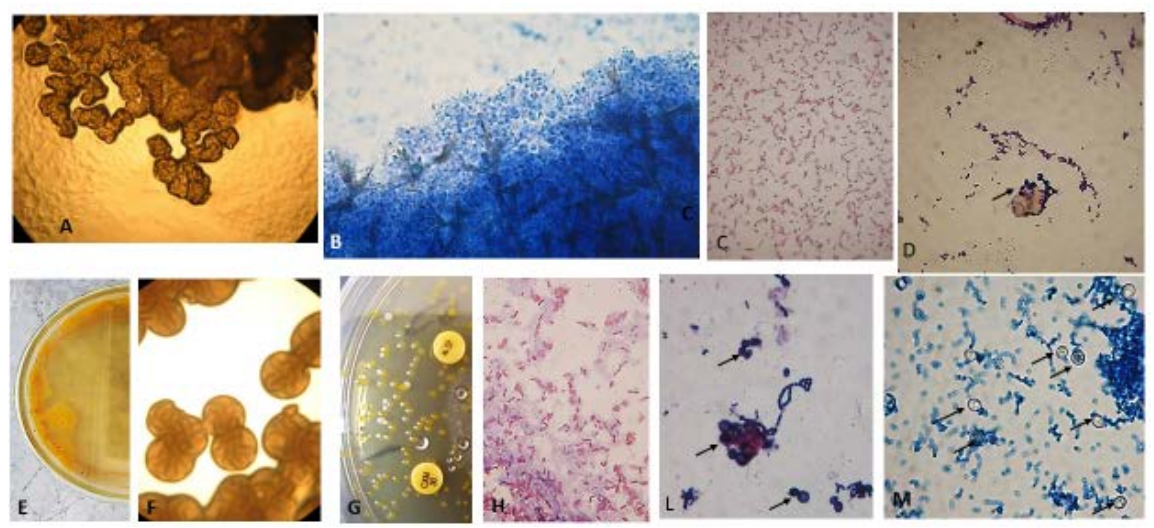

Figure 8. Co-isolation of communities of associated bacteria and fungi: Patient 4 (A, B, C and D); Mixed rough growth (A) and native preparation from the growth (B) consisting strongly associated bacteria and yeasts (Pseudomonas putida, Enterococcus faecalis and Rhodotorula mucilaginosa); Gram stained smear-Pseudomonas putida and Enterococcus faecalis (C); Gram stained smear-Enterococcus faecalis and Rhodotorula mucilaginosa (D); Patient 6 (E, F, G) Mixed rough growth (E) and rough colonies (F) consisting Pseudomonas luteola, Enterobacter cloacae and Rhodotorula mucilaginosa; smooth colonies of Pseudomonas luteola (G); Gram stained smear of Pseudomonas luteola (H); Patient 1-Gram stained smear of Streptococcus mitis and Rhodotorula mucilaginosa (L); Patient 3-Gram stained smear of Acinetobacter johnsonii and Rhodotorula mucilaginosa (M). Magnification: (B, C, D, H, L, M) - 1000×. (A, F)-200×.

inflammations anywhere in the body. In the context of chronically infected upper respiratory tract, of significance is to understand whether a local infection can be a source for entry of bacteria and fungi in the blood. On the other hand, it's of interest to study how bacteria and fungi transform into cell wall-deficient variants (L-forms) and in turns, how they can be detected and identified by isolation from blood.

The investigated cases in the current study include people of different age and sex with inflammation in the upper respiratory tract, varying in clinical symptoms, grade and chronicity of manifestations, as well as additional complications in the context of other diseases. A common feature that was noted concerning the blood microbiota of all investigated patients except the control healthy person, was a phenomenon of co-isolation of strongly/close associated bacterial and fungal cultures. It became clear, that the concrete combination of microbial species in every person (unique individual microbiota) can be considered as responsible for the variations in the manifestations of upper respiratory tract inflammations. It should be noted that in contrast to acute infection in upper respiratory tract when the etiological microbe agent can be easily diagnosed, in the cases with chronic infections this is difficult to be done. It's important in the chronic cases to detect which of microbial agents are able to overcome mucous barrier and to entry into blood circulation that in turns determinates its pathogenic effect in maintenance of the local infection. In this sense, it is of significance to analyze the data for each patient individually to assess his/her individual blood microbiota in correspondence to a persisting chronic local infection/in- 
flammation anywhere in the body.

From the blood of the first patient of age 7y.o., diagnosed with "hypertrophied adenoids", were isolated L-forms of bacteria-Streptococcus mitis/orales, Roseomonas mucosa and Dermacoccus nishinomiyaensis, together with fungiRhodotorula mucilaginosa and Aspergillus fumigatus. In particular, this combination of bacteria and fungi indicated that they originate/entered in blood from the upper respiratory tract (oro- and nasopharynx), that correlates adequately to the inflammation associated with "hypertrophied adenoids" in the patient. The "adenoids", are a part of the lymphoid tissue that naturally serve as a defense against infection. When its defense mechanisms overcome, it may become a site of acute and chronic infection [17].

In the second patient of age 32 y.o., with diagnosis chronic sinusitis, from the blood were isolated L-forms of Enterococcus faecalis and fungal species-Rhodotorula mucilaginosa, Mucorales and Aspergillus niger. Observation of elements from the life cycle of yeast and filamentous fungi was a leading finding. The final isolation of cultures on solid medium confirmed the vitality of the observed elements in the liquid medium in which the blood was initially incubated. This patient has been also diagnosed additionally with symptoms of migraine, although the headaches and nasal discharge are the most frequent symptoms in a chronic sinusitis [18]. The combination of life cycle elements from several fungal species is not surprising. Their detection in the blood does not mean that it is a systemic fungal infection, but rather a systemic entry of fungal elements into the bloodstream from some chronic focus of colonization/infection. It can be seen in the early stage of the study (Figure 1) how the cells of the immune system (polymorphonuclear and mononuclear leukocytes) phagocytized fungal spores. It should be noted that when culturing the blood of patient 2 in broth, large amounts of "mucoid secretion" were observed (Figure 7). This phenomenon can be associated with ability of Mucorales to secrete extracellular substances (polysaccharides), which probably contribute to the pathogenesis of sinusitis (soft tissue edema, disturbed drainage of secretions, etc.). Enterococcus faecalis is a bacterial species that is very easily transformed into L-forms, entering the bloodstream. In this case, Enterococcus faecalis is present in the blood asymptotically as L-forms. It is supposed that this species may contribute to the chronicity of inflammation caused by other microbes [19].

In the third patient of age 34y.o. with diagnoses nasal polyps, asthma bronchiale and allergic skin rash, were isolated from the blood L-forms of Acinetobacter johnsonii and fungal species-Rhodotorula mucilaginosa and Aspergillus fumigatus. Nasal polyps are most often associated with allergic diseases [20]. Acinetobacter johnsonii is defined as an opportunistic bacterial species that can cause infections ranging from pneumonia to bacteremia, urinary tract and soft tissue infections. A case of skin and soft tissue infection associated with previous antibiotic use has also been reported [21].

In the fourth patient of age 68y.o., with diagnoses chronic sinusitis and cer- 
vical lymphadenopathy as complication, were isolated from the blood Enterococcus faecalis, Pseudomonas putida and Rhodotorula mucilaginosa. An interesting feature in this case was the polymicrobial growth of co-aggregated cultures, what in turns was in correspondence to the granulomatous type of infection found in a cervical lymph node. Pseudomonas putida is a saprophytic species, but under certain conditions can be opportunistic pathogen. It has been reported to be isolated from patient with nasal necrosis [22].

In the fifth patient of age 32y.o., with diagnosis chronic nasopharyngitis, from the blood were isolated L-forms of Staphylococcus aureus and Aspergillus fumigatus. These microbial agents probably enter in the blood from a chronic infectious focus with localization in the nasopharynx. Staphylococcus aureus is a pathogenic bacterial species that generally causes purulent infections associated with various virulence factors. However, in this case it deals about L-forms of Staphylococcus aureus that are less pathogenic, but they retain the ability to produce toxins and are the cause of symptoms characteristic for the chronic infections.

The leading finding of clinical significance in patient 6 of age 62 y.o., with chronic upper respiratory tract infection complicated with "round pneumonia", was the combination of the two bacterial species Pseudomonas luteola and Enterobacter cloacae with yeast of species Rhodotorula mucilaginosa. Although these microbial species were not isolated from sputum by conventional methods, the presence of their L-forms in the blood of the patient suggest about their possible etiological role in causing the lung infection. Similar combination of Pseudomonas luteola and Escherichia vulneris has been reported to cause "round pneumonia", in which the lesion has been detected on the chest radiographs as solitary lung nodule, which are first suspected to be a lung cancer [23]. Infections with Pseudomonas luteola are less common, but cases of bacteremia, empyema, endocarditis, post-surgical infections and peritonitis have been reported [24]. Enterobacter cloacae is involved in the normal intestinal microbiota in many people. However, under certain conditions (immunosuppression), it can manifest itself as an opportunistic pathogen and cause a number of infections such as bacteremia, lower respiratory tract infection, urinary tract infections, endocarditis and others [25]. Findings related to the presence in the blood of elements of the life cycle of yeast and isolation of Rhodotorula mucilagenosa are rather an indicator of a state of suppressed immunity and entering of fungal elements probably from upper respiratory tract into the bloodstream without to claim about systemic fungal infection.

The mucosa in upper respiratory tract play an indispensable role to counter nominal pathogenic microbial colonization and as a barrier against microbial entry into blood circulation [25].

\section{Conclusion}

In conclusion, the novel innovative methodology for assessment of blood L-form 
microbiota was successfully applied for detection and evaluation of microbial agents responsible for chronic infections at upper respiratory tract.

\section{Conflicts of Interest}

The author declares no conflicts of interest regarding the publication of this paper.

\section{References}

[1] Markova, N. (2017) L-Form Bacteria Cohabitants in Human Blood: Significance for Health and Diseases. Discovery Medicine, 128, 305-313.

[2] Damgaard, C., Magnussen, K., Enevold, C., Nilsson, M., Tolker-Nielsen, T., Holmstrup, P. and Nielsen, C.H. (2015) Viable Bacteria Associated with Red Blood Cells and Plasma in Freshly Drawn Blood donations. PLOS ONE, 10, e0120826. https://doi.org/10.1371/journal.pone.0120826

[3] Dinakaran, V., Rathinavel, A., Pushpanathan, M., Sivakumar, R., Gunasekaran, P. and Rajendhran, J. (2014) Elevated Levels of Circulating DNA in Cardiovascular Disease Patients: Metagenomic Profiling of Microbiome in the Circulation. PLoS ONE, 9, e105221. https://doi.org/10.1371/journal.pone.0105221

[4] Domingue, G.J. and Schlegel, J.U. (1977) Novel Bacterial Structures in Human Blood: Cultural Isolation. Infection and Immunity Journal, 15, 621-627. https://doi.org/10.1128/iai.15.2.621-627.1977

[5] McLaughlin, R.W., Vali, H., Lau, P.C., Palfree, R.G., De Ciccio, A., Sirois, M., Ahmed, D., Vilemur, R., Desrosiers, M., Chan, E.C., et al. (2002) Are There Naturally Occurring Pleomorphic Bacteria in the Blood of Healthy Humans? Journal of Clinical Microbiology Journal, 40, 4771-4775. https://doi.org/10.1128/JCM.40.12.4771-4775.2002

[6] Moriyama, K., Ando, C., Tashiro, K., Kuhara, S., Okamura, S., Nakano, S., Takagi, Y., Miki, T., Nakashima, Y. and Hirakawa, H. (2008) Polymerase Chain Reaction Detection of Bacterial 16S rRNA Gene in Human Blood. Microbiology and Immunology, 52, 375-382. https://doi.org/10.1111/j.1348-0421.2008.00048.x

[7] Nikkari, S., McLaughlin, I.J., Bi, W., Dodge, D.E. and Relman, D.A. (2001) Does Blood of Healthy Subjects Contain Bacterial Ribosomal DNA? Journal of Clinical Microbiology Journal, 39, 1956-1959. https://doi.org/10.1128/JCM.39.5.1956-1959.2001

[8] Païssé, S., Valle, C., Servant, F., Courtney, M., Burcelin, R., Amar, J. and Lelouvie, B. (2016). Comprehensive Description of Blood Microbiome from Healthy Donors Assessed by 16S Targeted Metagenomic Sequencing. Transfusion, 56, 1138-1147. https://doi.org/10.1111/trf.13477

[9] Tedeschi, G., Amici, D. and Paparelli, M. (1969) Incorporation of Nucleosides and Amino-acids in Human Erythrocyte Suspensions: Possible Relation with a Diffuse Infection of Mycoplasms or Bacteria in the L-Form. Nature, 222, 1285-1286. https://doi.org/10.1038/2221285a0

[10] Markova, N., Slavchev, G. and Michailova, L. (2015) Presence of Mycobacterial L-Forms in Human Blood: Challenge of BCG Vaccination. Human Vaccines \& Immunotherapeutics, 11, 1192-1200. https://doi.org/10.1080/21645515.2015.1016682

[11] Markova, N., Slavchev, G., Djerov, L., Nikolov, A. and Dimova, T. (2016) Mycobacterial L-Forms Are Found in Cord Blood: A Potential Vertical Transmission of BCG 
from Vaccinated Mothers. Human Vaccines \& Immunotherapeutics, 12, 2565-2571. https://doi.org/10.1080/21645515.2016.1193658

[12] Dimova, T., Terzieva, A., Djerov, L., Dimitrova, V., Nikolov, A., Grozdanov, P. and Markova, N. (2017) Mother-to-Newborn Transmission of Mycobacterial L-Forms and V82 T-Cell Response in Placentobiome of BCG-Vaccinated Pregnant Women. Scientific Reports, 7, Article No. 17366. https://doi.org/10.1038/s41598-017-17644-Z

[13] Markova, N. (2019) Dysbiotic Microbiota in Autistic Children and Their Mothers: Persistence of Fungal and Bacterial Wall-Deficient L-Form Variants in Blood. Scientific Reports, 9, Article No. 13401. https://doi.org/10.1038/s41598-019-49768-9

[14] Markova, N. (2020) Eubiotic vs. Dysbiotic Human Blood Microbiota: The Phenomenon of Cell Wall Deficiency and Disease-Trigger Potential of Bacterial and Fungal L-Forms. Discovery Medicine, 156, 17-26.

[15] Potgieter, M., Bester, J., Kell, D.B. and Pretorius, E. (2015) The Dormant Blood Microbiome in Chronic, Inflammatory Diseases. FEMS Microbiology Reviews, 39, 567-591. https://doi.org/10.1093/femsre/fuv013

[16] Singhal, N., Kumar, M., Kanaujia, P.K. and Virdi, J.S. (2015) MALDI-TOF Mass Spectrometry: An Emerging Technology for Microbial Identification and Diagnosis. Frontiers Microbiology, 6, Article No. 791. https://doi.org/10.3389/fmicb.2015.00791

[17] Wang, H. (2020) Chronic Adenoids. Journal of International Medical Research, 48, Article ID: 0300060520971458. https://doi.org/10.1177\%2F0300060520971458

[18] Hoggard, M., Wagner, B.W., Mackenzie, B., Jain, R., Taylor, M.W., Biswas, K. and Doug las, R.G. (2017) Chronic Rhinosinusitis and the Evolving Understanding of Microbial Ecology in Chronic Inflammatory Mucosal Disease. Clinical Microbiology Reviews Journal, 30, 321-348. https://doi.org/10.1128/CMR.00060-16

[19] Fiore, E., Van Tyne, D. and Gilmore, M.S. (2019) Pathogenicity of Enterococci. Microbiology Spectrum, 7, Article No. 1128. https://doi.org/10.1128/microbiolspec.GPP3-0053-2018

[20] Stevens, W.W., Schleimer, R.P. and Kern, R.C. (2016) Chronic Rhinosinusitis with Nasal Polyps. The Journal of Allergy and Clinical Immunology: In Practice, 4, 565572. https://doi.org/10.1016/j.jaip.2016.04.012

[21] Henao-Martínez, A.F., González-Fontal, G.R. and Johnson, S. (2012) A Case of Community-Acquired Acinetobacter junii-johnsonii Cellulitis. Biomedica, 32, 179 181. https://doi.org/10.7705/biomedica.v32i2.652

[22] Tilak, A.M., Bishop, J., Jeyarajan, H. and Grayson, J. (2020) Bacteria-Induced Nasal Necrosis with Negative Cultures. BMJ Case Reports CP, 13, Article No. e235778. https://doi.org/10.1136/bcr-2020-235778

[23] Nichols, J., Srisung, W. and Yarbrough, S. (2015) Round Pneumonia with Pseudomonas luteola and Escherichia vulneris Bacteremia. SWRCCC, 3, 15-17. https://doi.org/10.12746/swrccc2015.0312.153

[24] Bayhan, G.I., Senel, S., Tanir, G. and Ozkan, S. (2015) Bacteremia Caused by Pseudomonas luteola in Pediatric Patients. Japanese Journal of Infectious Diseases, 68, 50-54. https://doi.org/10.7883/yoken.JJID.2014.051

[25] Baraniuk, J. (1994) Physiology of Sinusitis. In: Druce, H.M., Ed., Sinusitis: Pathophysiology and Treatment, Vol. 1. Marcel Dekker, New York, 19-39. 


\section{List of Abbreviations}

URT: Upper respiratory tract

MALDI-TOF MS: Matrix assisted laser desorption ionization-time of flight mass spectrometry technology 